\title{
FÓRUM INTERNACIONAL DE PEDAGOGIA DO ESPORTE
}

\author{
Resumos simples
}

SUMÁRIO

\section{Painéis em Pedagogia do Esporte}

\section{Seção: Desenvolvimento de Treinadores Esportivos}

A afetividade no processo de ensino-aprendizagem esportiva: as relações professor-saberaluno

A epistemologia da prática profissional de treinador e treinadora: um olhar aos aspectos didático-metodológicos no futebol.

A prática da reflexão para a aprendizagem de treinadores esportivos: uma proposta para

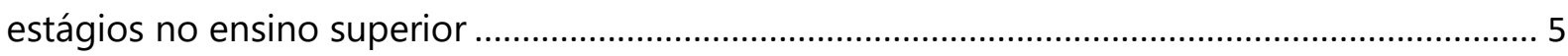

A relação interpessoal treinador/a-atleta no contexto do esporte escolar extracurricular ........ 6 capacitação de treinadores e treinadoras:................................................................................... 7

Um recurso necessário para o desenvolvimento do futebol de mulheres .................................. 7 Como deve agir o/a treinador/a que eu quero ser? Dilemas pedagógico-morais em meio às competições infantis.

Conhecimentos necessários para a intervenção profissional de treinadores de ginástica artística

Contextos e situações de aprendizagem de treinadoras(es) de handebol no Brasil...... 10

Desenvolvimento de um instrumento de medida do desenvolvimento positivo de idosos no esporte

Formação de treinadores(as) na universidade e o ensino centrado no(a) aprendiz: um estudo no curso de bacharelado em ciências do esporte da Unicamp.

Impacto da pandemia de covid-19 em aspectos psicológicos de atletas profissionais

O processo de "tornar-se" treinadora de surfe: análise de uma trajetória de participação social

Programa de formação de treinadores voltado ao ensino de habilidades para vida (HV) através do esporte. 


\section{Seção: Metodologia para o Ensino dos Esportes Coletivos}

Análise do basquete $3 \times 3$ e basquete de rua por meio do referencial histórico-cultural da pedagogia do esporte. 16

Investigação da sensibilidade pedagógica do treinador no futebol: um estudo exploratório 17 Caracterización de las demandas físico-fisiológicas según experiencia y métodos de enseñanza en baloncesto escolar. 18

Esporte contemporâneo: a pedagogia do esporte aplicada ao tchouckball 19 Influencia de las variables pedagógicas en la carga de entrenamiento en baloncesto en formación.

Influencia del cambio de sistema puntuación en la metodología de entrenamiento en patinaje artístico

As possibilidades e potencialidades técnico-táticas do jogo/brincadeira de bobinho em jovens futebolistas 23

O ensino-aprendizagem do futsal: um olhar para a educação básica e o ensino superior 24

\section{Seção: Percurso de Jovens no Esporte}

Sistema para el análisis las variables organizativas, pedagógicas y metodológicas en educación fisica

Variabilidad de la carga externa e interna atendiendo a la situación de juego en el desempeño del fútbol escolar. 26

Currículo da formação de jovens futebolistas: a iniciação esportiva 27

Desenvolvimento de atletas: a influência da qualidade da dinâmica social 28

Do esporte para a vida contribuições da pedagogia do esporte e do desenvolvimento positivo de jovens para a formação do cidadão

Esporte no contexto escolar: cenário das publicações em periódicos brasileiros de 1990 a 2019

Gestão em pedagogia do esporte: um estudo de caso em projeto social esportivo. 31

Desenvolvimento de habilidades para a vida por meio do rugby 32 


\title{
A AFETIVIDADE NO PROCESSO DE ENSINO-APRENDIZAGEM ESPORTIVA: AS RELAÇÕES PROFESSOR-SABER-ALUNO
}

\author{
Arthur William Santos Pinheiro \\ https://orcid.org/0000-0003-1985-624X \\ http://lattes.cnpq.br/5393970211356277 \\ Universidade Estadual de Campinas (Campinas, SP - Brasil) \\ a261782@dac.unicamp.br \\ Alcides José Scaglia \\ http://orcid.org/0000-0003-1462-1783 \\ http://lattes.cnpq.br/6052868681786447 9 \\ Universidade Estadual de Campinas (Campinas, SP - Brasil) \\ alcides.scaglia@fca.unicamp.br
}

Link do Painel: https://www.youtube.com/watch?v=K26DBUnZAJs\&list=PLQfgfDuqS1sYDDWE8aVpRSp-

J9JK5fkl\&index $=10$

\section{Resumo}

Introdução: O processo de ensino-aprendizagem esportiva envolve aspectos cognitivos, motores e afetivos, sendo o último de extrema importância, pois envolve a motivação, as paixões e emoções. Outro ponto importante desse processo é o das relações entre os sujeitos e o objeto a ser aprendido, no caso o esporte. Para a área da Didática, da Pedagogia do Esporte e da Psicologia da aprendizagem essas questões são extrema valia e podem ser objetos de estudos que contribuiriam para a formação de professores(as)/treinadores(as) com uma melhor sensibilidade pedagógica. Objetivo: Assim, o projeto visa investigar o processo de ensino-aprendizagem esportiva em "escolinhas" de basquetebol e a relevância ou caráter da afetividade como elemento essencial da formação do ser humano nesses ambientes. Além disso, analisar as relações didática, pedagógica e de aprendizagem em sessões de aulas/treinos nessas escolinhas levando em consideração a percepção dos alunos(as)/atletas sobre a conduta do professor(a)/treinador(a) quanto aos aspectos pedagógicos e afetivos.

Palavras-chave: Afetividade; Ensino-aprendizagem; Professor(a)/Treinador(a); Aluno(a)/Atleta; Esportes. 


\title{
A EPISTEMOLOGIA DA PRÁTICA PROFISSIONAL DE TREINADOR E TREINADORA: UM OLHAR AOS ASPECTOS DIDÁTICO- METODOLÓGICOS NO FUTEBOL
}

\author{
Luís Felipe Nogueira Silva \\ https://orcid.org/0000-0003-0583-4445 (iD \\ http://lattes.cnpq.br/0793380850491870 \\ Universidade Estadual de Campinas (Campinas, SP - Brasil) \\ luisfelipenogu@gmail.com \\ Alcides José Scaglia \\ http://orcid.org/0000-0003-1462-1783 \\ http://lattes.cnpq.br/6052868681786447 \\ Universidade Estadual de Campinas (UNICAMP) (Campinas, SP - Brasil) \\ alcides.scaglia@fca.unicamp.br \\ Link do Painel: https://www.youtube.com/watch?v=bcR-gWKI3CY\&list=PLJQfgfDuqS1sYDDWE8aVpRSp- \\ $\underline{\text { J9JK5fkl\&index }=8}$ \\ Introdução: As epistemologias da prática profissional compõem parte relevante das investigações científicas \\ voltadas ao desenvolvimento de treinadores e treinadoras de esportes que tome em conta a interação entre teorias \\ e práticas aplicadas e o alinhamento lógico entre métodos e didáticas. A subárea da Pedagogia do Esporte, ao \\ propor a organização, sistematização, aplicação e avaliação de conteúdos relevantes, sugere transcender as \\ dimensões biológicas e mecanicistas de atividades esportivas pela estruturação de modelos de ensino dinâmicos e \\ sistêmicos. Objetivo: Assim, o projeto visa desvelar a manifestação das epistemologias da prática profissional de \\ treinadores e treinadoras de futebol, no contexto de alto rendimento e apurar como a condução pedagógica, \\ metodológica e didática nos treinamentos reverberam modelos de ensino específicos à Pedagogia do Esporte. \\ Além disso, terão de treinamento observadas, de modo a terem examinadas suas relações com a práxis, a \\ constituição e mobilização de saberes profissionais e se obtenha um de suas identidades epistemológicas.
}

\section{Resumo}

Palavras-chave: Epistemologia; Treinador; Treinadora; Futebol; Saberes Profissionais. 


\title{
A PRÁTICA DA REFLEXÃO PARA A APRENDIZAGEM DE TREINADORES ESPORTIVOS: UMA PROPOSTA PARA ESTÁGIOS NO ENSINO SUPERIOR
}

\author{
Paula Simarelli \\ https://orcid.org/0000-0003-2527-7367 \\ http://lattes.cnpq.br/9378123927796564 9 \\ Universidade Estadual de Campinas (Campinas, SP - Brasil) \\ paula.simarelli@gmail.com \\ Michel Milistetd \\ https://orcid.org/0000-0003-3359-6878 \\ http://lattes.cnpq.br/3003314961521718 \\ Universidade Federal de Santa Catarina (Florianópolis, SC - Brasil) \\ michel.milistetd@ufsc.br \\ Roberto Rodrigues Paes \\ https://orcid.org/0000-0002-9333-9294 \\ http://lattes.cnpq.br/8646834864326813 \\ Universidade Estadual de Campinas (Campinas, SP - Brasil) \\ robertopaes@fef.unicamp.br
}

Link do Painel: https://www.youtube.com/watch?v=CQzkmf-Yg c\&list=PLJQfgfDuqS1sYDDWE8aVpRSp-

J9JK5fkl\&index $=2$

\begin{abstract}
Resumo
Introdução: A reflexão, elemento chave para a transformação de experiências em conhecimento, vem sendo defendida como importante prática ao desenvolvimento de um pensamento crítico e, consequente aprendizagem de treinadores esportivos. No Brasil, o estágio em Educação Física é entendido como momento potencial por se aproximar da lógica da aprendizagem experiencial, contrariando a lógica do acúmulo de conhecimentos na formação. Objetivo: Analisar as contribuições do desenvolvimento de práticas reflexivas para a aprendizagem e construção de um pensamento crítico-reflexivo de estudantes-treinadores em período de estágio. Metodologia: À luz da pesquisa-ação, serão realizadas intervenções e coletas ao longo de dois semestres. Serão realizadas entrevistas individualizadas e grupos focais com os estudantes no início, meio e final do processo. Os instrumentos reflexivos utilizados pelos alunos também servirão como fonte de dados. Resultados esperados: A partir da análise temática dos múltiplos dados, esperamos que os resultados contribuam com a formação de treinadores reflexivos ainda no período de ensino superior.
\end{abstract}

Palavras-chave: Reflexão; Aprendizagem Experiencial; Treinadores Esportivos; Estágio. 


\title{
A RELAÇÃO INTERPESSOAL TREINADOR/A-ATLETA NO CONTEXTO DO ESPORTE ESCOLAR EXTRACURRICULAR
}

\author{
Mayara de Almeida Tavares \\ https://orcid.org/0000-0002-2805-4276 \\ http://lattes.cnpq.br/5210341623824070 \\ Universidade do Estado de Mato Grosso (Cáceres, MT - Brasil) \\ mayara_tavares273@hotmail.com \\ Riller Silva Reverdito \\ https://orcid.org/0000-0003-0556-9151 \\ http://lattes.cnpq.br/3357837391641002 9 \\ Universidade do Estado de Mato Grosso (Cáceres, MT - Brasil) \\ rsreverdito@unemat.br
}

Link do Painel: https://www.youtube.com/watch?v=6cPQNJO9YUA\&list=PLJQfgfDuqS1sYDDWE8aVpRSp-

J9JK5fkl\&index $=4$

\begin{abstract}
Resumo
Introdução: As relações interpessoais são inevitáveis e as condutas desenvolvidas pelo/a treinador/treinadora podem influenciar de maneira significativa as experiências positivas e/ou negativas dos jovens atletas. Objetivo: Diante disso, o objetivo desse estudo é analisar a relação interpessoal de treinadores/treinadora-atleta com melhor relação interpessoal percebida pelas atletas no contexto escolar extracurricular. Metodologia: Trata-se de um estudo realizado com 3 treinadores/treinadora (Mas. $n=2$ - Fem. $n=1$ ) de diferentes regiões esportivas do estado de Mato Grosso e suas respectivas equipes, totalizando 20 jovens atletas do sexo feminino, com idade entre 15 e 17 anos, praticantes das modalidades handebol $(n=2)$ e basquetebol $(n=1)$. O conjunto de dados foi coletado no contexto de treino, através de entrevista semiestruturada com as atletas por meio do grupo focal. Resultado: Como resultado identificamos que os elementos que contribuem para qualidade das relações interpessoais positivas dos/da treinadores/treinadora com as atletas estão relacionados a confiança, amizade, afeto, cuidado e apoio social. Palavras-chave: Relação Interpessoal Treinador-Atleta; Jovens Atletas; Pedagogia do Esporte.
\end{abstract}

Agradecimentos: Fundação de Amparo à Pesquisa do Estado de Mato Grosso (FAPEMAT), Rede Cedes Mato Grosso. 


\title{
CAPACITAÇÃO DE TREINADORES E TREINADORAS: UM RECURSO NECESSÁRIO PARA O DESENVOLVIMENTO DO FUTEBOL DE MULHERES
}

\author{
Júlia Barreira \\ https://orcid.org/0000-0002-8065-4359 \\ http://lattes.cnpq.br/2462983373432879 \\ Universidade Estadual de Campinas (Campinas, SP - Brasil) \\ jubarreira2@hotmail.com \\ Larissa Rafaela Galatti \\ https://orcid.org/0000-0003-1743-6356 \\ http://lattes.cnpq.br/3409947437523352 \\ Universidade Estadual de Campinas (Campinas, SP - Brasil) \\ lgalatti@unicamp.br
}

Link do Painel: https://www.youtube.com/watch?v=hkfxgpgkYuM\&list=PLJQfgfDuqS1sYDDWE8aVpRSp$\underline{\text { J9JK5fkl\&index }=6}$

\section{Resumo}

Objetivo: O objetivo desse estudo foi identificar os fatores que fomentam ou dificultam o desenvolvimento do futebol de mulheres por uma perspectiva da gestão do esporte. Metodologia: Foram entrevistados 17 agentes de onze países que apresentam elevados níveis de participação e desempenho na modalidade. Resultado: A partir da análise temática, encontramos os Estados Unidos como o país de o maior protagonismo na modalidade. O seu desenvolvimento está atrelado, entre outros fatores, a uma mudança organizacional no oferecimento de cursos para profissionais de comissão técnica e exigência de formação para atuar na modalidade. Os demais países apresentam um processo de treinamento ainda dependente de familiares e com uma baixa exigência de qualificação. Conclusão: Nossos achados mostram que a qualificação de treinadores e treinadoras é um elemento fundamental para o desenvolvimento do futebol de mulheres e indicam a necessidade de uma maior participação de organizações esportivas nesse processo.

Palavras-chave: Desenvolvimento do Esporte; Formação; Gestão; Comissão Técnica. 


\title{
COMO DEVE AGIR O/A TREINADOR/A QUE EU QUERO SER? DILEMAS PEDAGÓGICO-MORAIS EM MEIO ÀS COMPETIÇÕES INFANTIS
}

\author{
Lucas Leonardo \\ https://orcid.org/0000-0002-1567-0686 \\ http://lattes.cnpq.br/0543067155062208 \\ Universidade Estadual de Campinas (Campinas, SP - Brasil) \\ prof.lucasleonardo@gmail.com \\ Alcides José Scaglia \\ http://orcid.org/0000-0003-1462-1783 \\ http://lattes.cnpq.br/6052868681786447 9 \\ Universidade Estadual de Campinas (Campinas, SP - Brasil) \\ alcides.scaglia@fca.unicamp.br
}

Link do Painel: https://www.youtube.com/watch?v=v4R6HJEAckE\&list=PLJQfgfDuqS1sYDDWE8aVpRSp-

$\underline{\text { JjJKfkl\&index }=5}$

\begin{abstract}
Resumo:
Introdução: Treinadores/as de handebol que transgredirem e subvertem regras de competições infantis são sempre imorais? Assumo para este debate, a partir de Paul Ricoeur, para quem ética e moral representam correntes opostas, mas complementares. Opostas, pois a ética é a estima do que é bom para a boa vida baseada nas singularidades e a moral é a imposição do que é obrigatório diante do sentimento de dever universal. Complementares, pois: a) Há primazia ética sobre a moral; b) Existe necessidade de intenção ética passar pelo crivo da norma; e c) diante de conflitos em que a moral cria impasses, é necessário recurso da moral à intenção ética. Objetivo: Compreender, do ponto moral em articulação com a da pedagogia do esporte, os motivos que levam treinadores/as à transgressão e subversão das regras do handebol infantil modificado, considerando seus quereres e deveres à luz de uma teoria ética dialógica. Resultados Esperados: Encontrar subsídios empíricos que possam ampliar o debate sobre o que é a moralidade do/a treinador/a, levando ao debate à possibilidade de que as atitudes transgressores e subversivas possam sustentar potencial pedagógico se alicerçadas pela solicitude e pelo respeito à criança em consonância com o conceito de momento pedagógico de Phillippe Meirieu. Espera-se, assim, encontrar possíveis contrapontos à hegemônica concepção de que é apenas o sentimento de amor de si o único motivador de tais condutas. Espero, com isso, abrir espaço para a superação da deontologia kantiana como único caminho de ajuizamento moral, estabelecendo a pequena ética de Paul Ricoeur como uma teoria filosófica de base para análise pedagógico-moral de treinadores e treinadoras por meio de uma via descendente de julgamento moral, esta, mais humanizante e menos apoiada na regra como dotada de um valor imperioso e indiscutível.
\end{abstract}

Palavras-chave: Moral; Ética; Esporte; Competição; Infância. 


\title{
CONHECIMENTOS NECESSÁRIOS PARA A INTERVENÇÃO PROFISSIONAL DE TREINADORES DE GINÁSTICA ARTÍSTICA
}

\author{
Lucas Machado de Oliveira \\ https://orcid.org/0000-0001-9229-1033 id \\ http://lattes.cnpq.br/0310115664031476 \\ Universidade Federal de Santa Catarina (Florianópolis, SC - Brasil) \\ lucasmdo@outlook.com \\ Vitor Ciampolini \\ http://orcid.org/0000-0003-2278-7310 iD \\ http://lattes.cnpq.br/9286890586590825 9 \\ Universidade Federal de Santa Catarina (Florianópolis, SC - Brasil) \\ vciampolini@gmail.com \\ Michel Milistetd \\ https://orcid.org/0000-0003-3359-6878 \\ http://lattes.cnpq.br/3003314961521718 \\ Universidade Federal de Santa Catarina (Florianópolis, SC - Brasil) \\ michel.milistetd@ufsc.br \\ Juliana Pizani \\ https://orcid.org/0000-0001-5489-1468 \\ http://lattes.cnpq.br/7149328880610490 \\ Universidade Federal de Santa Catarina (Florianópolis, SC - Brasil) \\ juliana.pizani@ufsc.br
}

Link do Painel: https://www.youtube.com/watch?v=qay3EvhvMpg\&list=PLQfgfDuqS1sYDDWE8aVpRSp-

$\underline{\text { J9JK5fkl\&index }=3}$

\begin{abstract}
Resumo
Objetivo: O estudo buscou mapear os conhecimentos necessários na atuação de treinadores de ginástica artística. Metodologia: Foram entrevistados cinco treinadores filiados à Federação de Ginástica de Santa Catarina, abordando indicadores como: rotina de atuação profissional e conhecimentos importantes nesse contexto. Utilizouse a Análise Temática de Braun e Clarke (2006) e a classificação de conhecimentos do treinador de Côté e Gilbert (2009) para o tratamento dos dados. Resultados: Os achados destacam a presença de conhecimentos profissionais na rotina dos treinadores, sobretudo a psicologia do esporte, pedagogia do esporte, gestão esportiva, treinamento esportivo e aspectos técnicos da modalidade. Conhecimentos interpessoais são identificados no relacionamento com atletas e pais e na mediação de conflitos fora do contexto esportivo. Com menor ênfase, o autoconhecimento e capacidade de refletir e avaliar a própria atuação são temas que indicam a presença de conhecimentos intrapessoais. Maiores investigações são necessárias para elucidar os conhecimentos intrapessoais na intervenção do treinador.
\end{abstract}

Palavras-chave: Conhecimentos do Treinador; Prática Profissional; Ginástica Artística. 


\title{
CONTEXTOS E SITUAÇÕES DE APRENDIZAGEM DE TREINADORAS(ES) DE HANDEBOL NO BRASIL
}

\author{
Matheus de Seixas Bizetti \\ https://orcid.org/0000-0003-2588-3420 \\ http://lattes.cnpq.br/4680393666700970 \\ Universidade Estadual de Campinas (Campinas, SP - Brasil) \\ mabizetti@gmail.com \\ Larissa Rafaela Galatti \\ https://orcid.org/0000-0003-1743-6356 \\ http://lattes.cnpq.br/3409947437523352 \\ Universidade Estadual de Campinas (Campinas, SP - Brasil)
}

Link do Painel: https://www.youtube.com/watch?v=SPRWbNamwZ0\&list=PLJQfgfDuqS1sYDDWE8aVpRSp-

J9JK5fkl\&index $=7$

\section{Resumo}

Objetivo: O projeto de pesquisa exploratória propõe-se a investigar a percepção de treinadoras(es) dos Acampamentos Nacionais de Desenvolvimento e Melhoria Técnica como um episódio de aprendizagem na formação destas(es) treinadoras(es). Metodologia: A primeira etapa desenvolvida pelo mapeamento de indivíduos que potencialmente foram impactados pelos acampamentos, análise documental dos Acampamentos, seguido de aplicação de questionários que auxiliam a melhor compreender este envolvimento. Após realização de projeto piloto, segue-se um conjunto de entrevistas semiestruturadas com coordenadoras(es) dos Acampamentos que contribuem no processo de compreensão do desenvolvimento do programa, assim como o estabelecimento dos centros de análise de agrupamento de treinadoras(es) que responderam ao questionário. Uma série de entrevistas semiestruturadas serão realizadas com representantes de cada agrupamento. Projeto em desenvolvimento, aplicação do projeto piloto prevista para os meses de Junho e Julho de 2021.

Palavras chave: Desenvolvimento de Treinadoras(es); Handebol; Acampamentos. 


\title{
DESENVOLVIMENTO DE UM INSTRUMENTO DE MEDIDA DO DESENVOLVIMENTO POSITIVO DE IDOSOS NO ESPORTE
}

\author{
Bartira Pereira Palma \\ https://orcid.org/0000-0002-7648-8060 ip \\ http://lattes.cnpq.br/5955792372543419 \\ Universidade Estadual de Campinas (Campinas, SP - Brasil) \\ bartirapalma@gmail.com \\ Larissa Rafaela Galatti \\ https://orcid.org/0000-0003-1743-6356 id \\ http://lattes.cnpq.br/3409947437523352 9 \\ Universidade Estadual de Campinas (Campinas, SP - Brasil) \\ Igalatti@unicamp.br \\ Link do Painel: https://www.youtube.com/watch?v=W8RtGvdGkLU\&list=PLJQfgfDuqS1sYDDWE8aVpRSp- \\ J9JK5fkl\&index $=11$

\begin{abstract}
Resumo
Introdução: O contexto esportivo pode promover o desenvolvimento de potencialidades humanas, o que ainda é pouco explorado em idosos. Objetivo: O objetivo deste estudo é desenvolver um instrumento para avaliação do desenvolvimento positivo de idosos por meio do esporte no contexto brasileiro. Metodologia: Será realizada revisão sistemática da literatura para identificar instrumentos de medida do desenvolvimento positivo e compreender os modelos teóricos utilizados. Então, serão realizados grupos focais com 48 idosos praticantes de esporte e entrevistas com cerca de 6 treinadores esportivos de idosos a fim de se identificar expressões dos construtos específicos para esse contexto. Após, será desenvolvido o instrumento de medida que passará por processo de avaliação das propriedades psicométricas. Duzentos idosos praticantes de esporte responderão ao instrumento, que será analisado quanto à estrutura interna, consistência interna e fidedignidade. Resultado esperado: Espera-se desenvolver um instrumento com evidências de validade e precisão que contribuirá com a prática profissional.
\end{abstract} \\ Palavras-chave: Competências Socioemocionais; Envelhecimento; Pedagogia do Esporte; Avaliação.
}




\title{
FORMAÇÃO DE TREINADORES(AS) NA UNIVERSIDADE E O ENSINO CENTRADO NO(A) APRENDIZ: UM ESTUDO NO CURSO DE BACHARELADO EM CIÊNCIAS DO ESPORTE DA UNICAMP
}

\author{
Yura Yuka Sato dos Santos \\ https://orcid.org/0000-0002-9422-6953 \\ http://lattes.cnpq.br/7508755808597936 \\ Universidade Estadual de Campinas (Campinas, SP - Brasil) \\ yura_sato@hotmail.com \\ Larissa Rafaela Galatti \\ https://orcid.org/0000-0003-1743-6356 \\ http://lattes.cnpq.br/3409947437523352 9 \\ Universidade Estadual de Campinas (Campinas, SP - Brasil) \\ lgalatti@unicamp.br
}

Link do Painel: https://www.youtube.com/watch?v=zpZQkdRGxQg\&list=PLJQfgfDuqS1sYDDWE8aVpRSp-

J9JK5fkl\&index $=1$

\section{Resumo}

Objetivo: O objetivo foi investigar, por meio da pesquisa-ação, o desenvolvimento do ensino centrado no aprendiz na formação universitária de treinadores(as), especificamente na disciplina Treinador Desportivo, do curso de Ciências do Esporte da UNICAMP, nos anos de 2018 em ambiente de ensino presencial, e 2020 em ambiente de ensino remoto emergencial. Metodologia: Participaram a pesquisadora, a professora, sete auxiliares docentes e estudantes-treinadores(as), que responderam a questionários e participaram de grupos focais. Os dados foram analisados pela análise temática. Os(as) estudantes-treinadores(as) perceberam uma aproximação da formação à realidade prática do(a) treinador(a). Em 2020, o foco no desenvolvimento de um ambiente propício à aprendizagem e no estímulo à assunção da responsabilidade pela aprendizagem, parece ter potencializado o desenvolvimento de habilidades reflexivas e de aprendizagem autodirigida dos(as) estudantes. O suporte docente aos discentes auxiliou a amenizar as dificuldades geradas pela pandemia e ensino remoto emergencial, de forma que os(as) estudantestreinadores(as) conseguissem seguir os estudos na disciplina.

Palavras-chave: Formação Inicial; Ensino Centrado no Aprendiz; Treinador; Treinadora. 


\title{
IMPACTO DA PANDEMIA DE COVID-19 EM ASPECTOS PSICOLÓGICOS DE ATLETAS PROFISSIONAIS
}

\author{
Alessandra Santos de Oliveira \\ https://orcid.org/0000-0001-8170-5122 \\ http://lattes.cnpq.br/9544900137258812 \\ Universidade São Judas Tadeu (São Paulo, SP - Brasil) \\ aleball13@gmail.com \\ Douglas Vinicius Carvalho Brasil \\ https://orcid.org/0000-0002-0797-6319 iD \\ http://lattes.cnpq.br/1944489828481383 \\ Universidade Estadual de Campinas (Campinas, SP - Brasil) \\ d138267@dac.unicamp.br \\ Nayara Malheiros Caruzzo \\ http://orcid.org/0000-0002-9356-3886 \\ http://lattes.cnpq.br/9293422406626348 \\ Universidade Estadual de Maringá, (Maringá, PR - Brasil) \\ nayaramalheiros@gmail.com

Maria Regina Brandão
https://orcid.org/0000-0001-9069-4672 iD
http://lattes.cnpq.br/9246570791969907
Universidade São Judas Tadeu (São Paulo, SP - Brasil)
mrfbrandao@gmail.com \\ Roberto Rodrigues Paes \\ https://orcid.org/0000-0002-9333-9294 \\ http://lattes.cnpq.br/8646834864326813 \\ Universidade Estadual de Campinas (Campinas, SP - Brasil) \\ robertopaes@fef.unicamp.br
}

Link do Painel: https://www.youtube.com/watch?v=hac5LR2RtAY\&list=PLJQfgfDuqS1sYDDWE8aVpRSp-

J9JK5fkl\&index $=12$

\section{Resumo}

Introdução: A COVID-19 se proliferou rapidamente ao redor do mundo, culminando em uma pandemia que afeta negativamente diversos campos da sociedade, dentre os quais os relacionados ao esporte. Objetivo: Na presente pesquisa buscaremos identificar de que modo esta crise sanitária tem afetado aspectos psicológicos de atletas e treinadores esportivos profissionais. Metodologia: Para tal, utilizaremos os questionários WHOQOL e DASS-21, os quais estão sendo aplicados em três momentos distintos a partir do início da pesquisa. Resultados Esperados: A partir dos resultados esperamos conseguir descrever o estado psicológico das voluntárias e voluntários durante esse período, de modo a auxiliar que atletas, treinadores e treinadoras, profissionais de Educação Física e Ciências do Esporte (dentre outros) inseridos no contexto esportivo busquem estratégias para amenizar possíveis efeitos negativos causados por crises como a que estamos vivendo.

Palavras-chave: Pedagogia do Esporte; Psicologia do Esporte; Educação Física; Ciências do Esporte; COVID-19. 


\title{
O PROCESSO DE “TORNAR-SE” TREINADORA DE SURFE: ANÁLISE DE UMA TRAJETÓRIA DE PARTICIPAÇÃO SOCIAL
}

\author{
Vinicius Zeilmann Brasil \\ https://orcid.org/0000-0003-0036-494X ip \\ http://lattes.cnpq.br/7488411738269363 \\ Universidade do Estado de Santa Catarina (Florianópolis, SC - Brasil) \\ vzbrasil@hotmail.com
}

Link do Painel: https://www.youtube.com/watch?v=aLwXN8VVCLE\&list=PLJQfgfDuqS1sYDDWE8aVpRSp-

$\underline{\text { J9JK5fkl\&index }=9}$

\begin{abstract}
Resumo
Introdução: A informalidade da popularização dos esportes de aventura e o caráter adaptativo da intervenção do treinador nestas modalidades, sugerem maior valorização das experiências esportivas e profissionais, no processo de tornar-se treinador. Objetivo: Analisou-se as implicações das experiências de uma treinadora de surfe para tornar-se treinadora. Metodologia: Realizou-se um estudo de caso com uma treinadora de surfe, combinando entrevista semiestruturada, observação participante e o Rappaport Time Line. Os dados foram analisados mediante a Análise Temática. Resultado: O engajamento da treinadora, desde a infância, em práticas ligadas ao lazer em família, ao esporte e ao surfe, permitiram a aquisição de competências, de valores e perspectivas, para se tornar surfista. Enquanto atleta e treinadora, a (re)negociação dos significados de suas experiências permitiu a consolidação de seus conhecimentos e perspectivas à intervenção profissional. Conclusão: Tornar-se treinadora abrangeu um processo contínuo de (re)construção dos significados, das competências e da identidade, em função da participação no contexto do surfe.
\end{abstract}

Palavras-chave: Esportes de Aventura; Surfe; Treinador Esportivo; Aprendizagem Social. 


\title{
PROGRAMA DE FORMAÇÃO DE TREINADORES VOLTADO AO ENSINO DE HABILIDADES PARA VIDA (HV) ATRAVÉS DO ESPORTE
}

\author{
Carlos Ewerton Palheta \\ https://orcid.org/0000-0001-7066-7562 id \\ http://lattes.cnpq.br/6996592239389013 \\ Universidade Federal de Santa Catarina (Florianópolis, SC - Brasil) \\ carlospalheta26@gmail.com \\ Michel Milistetd \\ https://orcid.org/0000-0003-3359-6878 \\ http://lattes.cnpq.br/3003314961521718 \\ Universidade Federal de Santa Catarina (Florianópolis, SC - Brasil) \\ michel.milistetd@ufsc.br
}

Link do Painel: https://www.youtube.com/watch?v=wHwXBrbfa c\&list=PLQfgfDuqS1sYDDWE8aVpRSp-

J9JK5fkl\&index $=13$

\begin{abstract}
Resumo
Introdução: Este trabalho apresenta a fase de elaboração de um programa de formação de treinadores para o ensino de HV em um clube multiesportivo. Em um ano, o programa teve a definição de diretrizes, construção de materiais pedagógicos, realização de workshop e monitoramento da intervenção em uma fase piloto de implementação. Metodologia: Entrevistas foram realizadas com quatro coordenadores e doze treinadores. 24 sessões de treinamento foram observadas com auxílio do PQAYS (BEAN et al., 2018) em dois momentos em um intervalo de três meses. Resultados: Resultados preliminares demonstram que a descrição de HV nas diretrizes do clube tem motivado os treinadores a ensinar HV. A interação entre os profissionais nos momentos de treinamento tem proporcionado aos treinadores a capacidade de ensinar HV. Definir o ensino de HV enquanto dever dos treinadores tem proporcionado a eles a oportunidade de alinhar suas perspectivas pessoais com a filosofia do clube ao qual pertencem.
\end{abstract}

Palavras-chave: Desenvolvimento Positivo de Jovens; Formação Continuada; Programa Esportivo. 


\title{
ANÁLISE DO BASQUETE 3X3 E BASQUETE DE RUA POR MEIO DO REFERENCIAL HISTÓRICO-CULTURAL DA PEDAGOGIA DO ESPORTE
}

\author{
Douglas Vinicius Carvalho Brasil \\ https://orcid.org/0000-0002-0797-6319 id \\ http://lattes.cnpq.br/1944489828481383 \\ Universidade Estadual de Campinas (Campinas, SP - Brasil) \\ d138267@dac.unicamp.br \\ Roberto Rodrigues Paes \\ https://orcid.org/0000-0002-9333-9294 iD \\ http://lattes.cnpq.br/8646834864326813 9 \\ Universidade Estadual de Campinas (Campinas, SP - Brasil) \\ robertopaes@fef.unicamp.br \\ Link do Painel:
}

https://www.youtube.com/watch?v=zhHeglalal0\&list=PLJQfgfDuqS1tDpTzZzgdLchBDvZa8SIAp\&index=3

\begin{abstract}
Resumo
Introdução: O "Referencial Histórico-Cultural da Pedagogia do Esporte" possibilita refletir acerca da criação e desenvolvimento das diferentes práticas esportivas, tendo potencial para contribuir com a formação crítica dos sujeitos e a compreensão do fenômeno esportivo para além de seus aspectos técnico-táticos. Objetivo: buscamos compreender o "Basquete de Rua" (BDR) e o "Basquete 3×3" (B3×3), por vezes compreendidos enquanto sinônimos por diferentes atores. Metodologia: Revisão bibliográfica, norteada por este referencial, Resultado: Evidenciamos que o B3x3 e o BDR possuem história, desenvolvimento e características distintas. Portanto, é um equívoco compreendê-los enquanto sinônimos. Conclusão: Por fim, consideramos que tais diferenças podem e devem ser abordadas em diferentes contextos de ensino, de modo que o processo de vivência e aprendizagem destas práticas corporais (a certa medida) contribua para que, quem as vivencie não se tornem meros reprodutores acríticos de técnicas, táticas, discursos e comportamentos.
\end{abstract}

Palavras-chave: Pedagogia do Esporte; Educação Física; Basquete 3×3; Basquete de Rua; Basquetebol. 


\title{
INVESTIGAÇÃO DA SENSIBILIDADE PEDAGÓGICA DO TREINADOR NO FUTEBOL: UM ESTUDO EXPLORATÓRIO
}

\author{
Alberto Lobato Góes Júnior \\ https://orcid.org/0000-0002-7421-3901 \\ http://lattes.cnpq.br/2963844434616048 9 \\ Universidade Estadual de Campinas (Campinas, SP - Brasil) \\ algj1421@gmail.com \\ João Bosco Gomes Lima Júnior \\ https://orcid.org/0000-0001-8315-730X \\ http://lattes.cnpq.br/4802924119461331 \\ Universidade Estadual de Campinas (Campinas, SP - Brasil) \\ bosco_junior@outlook.com \\ Alcides José Scaglia \\ http://orcid.org/0000-0003-1462-1783 \\ http://lattes.cnpq.br/6052868681786447 \\ Universidade Estadual de Campinas (Campinas, SP - Brasil) \\ alcides.scaglia@fca.unicamp.br \\ Link do Painel:
}

https://www.youtube.com/watch?v=w Zx BqBgUc\&list=PLQfgfDuqS1tDpTzZzgdLchBDvZa8SIAp\&index=6

\begin{abstract}
Resumo
Objetivo: Investigar a sensibilidade pedagógica do treinador. Metodologia: Participaram da pesquisa dois treinadores de futebol (A, B) pertencentes a categoria sub $13(n=10)$ e sub $17(n=10)$, sendo respeitados todos os procedimentos éticos. Cada treinador construiu duas atividades (Jogo1; Jogo2) relacionadas com o tema de progressão ao alvo. Foi empregado uma análise notacional por meio dos indicadores simples e compostos da performance. Na análise estatística, a normalidade foi avaliada pelo teste de Kolmogorovsmirnov e o teste de Mannwhitney para comparações intra-treinadores, foi utilizado o tamanho do efeito para a magnitude das diferenças, o nível de significância foi de $5 \%(p<0,05)$ e o tratamento estatístico foi feito no software GraphPad Prism. Resultado: As atividades do treinador A apontaram para a temática de manutenção da posse de bola, enquanto as do treinador B trabalharam o tema proposto. Consideração: Conclui-se que a sensibilidade pedagógica dos treinadores influenciou no tema original da aula.
\end{abstract}

Palavras-chave: Futebol; Jogo; Pedagogia do Jogo; Sensibilidade. 


\title{
CARACTERIZACIÓN DE LAS DEMANDAS FÍSICO-FISIOLÓGICAS SEGÚN EXPERIENCIA Y MÉTODOS DE ENSEÑANZA EN BALONCESTO ESCOLAR
}

\author{
María Graças Gamero \\ Universidad de Extremadura (Cáceres - España) \\ mgamero@alumnos.unex.es
}

Juan Manuel García-Ceberino https://orcid.org/0000-0002-2922-4935 iD Universidad de Extremadura, (Cáceres - España) jmanuel.jmgc@gmail.com

Sebastián Feu

https://orcid.org/0000-0003-2959-5960 id

Universidad de Extremadura, (Cáceres - España)

sfeu@unex.es

Link do Painel: https://www.youtube.com/watch?v=UIrrOzF94vU\&list=PLQfgfDuqS1tDpTzZzgdLchBDvZa8SIAp

\begin{abstract}
Resumo
Introducción. La metodología que utiliza el profesor de Educación Física en sus sesiones condiciona la aptitud física de los alumnos, pues implica cargas de trabajo diferentes. El objetivo del estudio fue cuantificar y comparar, en función del método de enseñanza y la experiencia previa del alumno, la carga externa (eTL) e interna (iTL) derivadas de la aplicación de tres programas de intervención basados en métodos de enseñanza diferentes, Tactical Games Aproach (TGA), Direct Instruction (DI) y Service Teacher's Basketball Unit (STBU) para la enseñanza del baloncesto escolar. Método. El presente estudio se encuadra dentro de una estrategia manipulativa de tipo cuasi-experimental y longitudinal. La muestra estuvo formada por 49 estudiantes de $6^{\circ}$ curso de educación primaria $\left(6^{\circ} \mathrm{A}, 6^{\circ} \mathrm{B}\right.$ y $\left.6^{\circ} \mathrm{C}\right)$. Las variables dependientes del estudio fueron las demandas físico-fisiológicas registradas por los estudiantes durante la intervención. Se registraron un total de 30 variables diferenciándose en eTL y iTL. Para registrar las variables eTL cada estudiante se equipó con un dispositivo inercial WIMU Pro TM (Real Track Systems, Almería, España), y para registrar las variables iTL se utilizó las bandas de frecuencia cardiaca GARMIN TM. Por último, se utilizó el software SPRO TM (Real Track Systems, Almería, España) para convertir la información en datos cuantitativos. La intervención tuvo una duración de 11 horas, nueve horas pertenecientes a los programas de intervención, y dos pertenecientes a las pruebas de evaluación (pre-test/post-test), en las que se disputaron partidos de 3 vs 3 . Resultados y discusión. Los resultados identificaron diferencias significativas a favor de los estudiantes que recibieron el programa basado en el método de enseñanza TGA. Durante la aplicación de los programas, los estudiantes del método TGA registraron valores más altos de eTL e iTL (frecuencia cardíaca media). Por tanto, el diseño de las tareas bajo diferentes enfoques repercute directamente en la carga de trabajo que experimentan los estudiantes. Durante las pruebas de evaluación, presentaron niveles de frecuencia cardíaca similares a los del resto de estudiantes; sin embargo, realizaron tareas de más intensidad utilizando con mayor frecuencia las velocidades correr y esprintar. Por tanto, presentaron mejor condición física. Durante las pruebas de evaluación, los estudiantes sin experiencia previa mostraron niveles más altos de velocidad máxima; mientras que los estudiantes con experiencia presentaron mayores niveles de frecuencia cardiaca. Esto podría deberse a que los estudiantes con experiencia trabajaron durante mayor tiempo en los rangos de alta intensidad. Conclusión. El método TGA registró valores más altos de eTL e iTL, favoreciendo la condición física y la salud de los estudiantes. Por tanto se recomienda su uso para la planificación de las sesiones de educación primaria.
\end{abstract}

Palabras clave: Métodos de Enseñanza; Tactical Games Aproach; Baloncesto Escolar.

\section{Agradecimientos}

Trabajo parcialmente subvencionado por la Ayuda a los Grupos de Investigación (GR18170) del Gobierno de Extremadura (Consejería de Economía, Ciencia y Agenda Digital); con la aportación de la Unión Europea a través de los Fondos Europeos de Desarrollo Regional. 


\title{
ESPORTE CONTEMPORÂNEO: A PEDAGOGIA DO ESPORTE APLICADA AO TCHOUCKBALL
}

\author{
Silas Sinotti \\ https://orcid.org/0000-0003-4054-4566 \\ http://lattes.cnpq.br/0668884556418155 9 \\ Universidade Estadual de Campinas (Campinas, SP - Brasil) \\ s234754@dac.unicamp.br \\ Roberto Rodrigues Paes \\ https://orcid.org/0000-0002-9333-9294 \\ http://lattes.cnpq.br/8646834864326813 \\ Universidade Estadual de Campinas (Campinas, SP - Brasil) \\ robertopaes@fef.unicamp.br
}

Link do Painel:

https://www.youtube.com/watch?app=desktop\&v=BBsDEu8SNw\&list=PLQfgfDuqS1tDpTzZzgdLchBDvZa8SIAp\&i

$\underline{\text { ndex }=2}$

\section{Resumo}

Introdução: Os esportes necessitam de uma estruturação pedagógica, metodológica, uma organização da forma de ensinar a estratégia, a tática, técnica, de modo a aprender a jogar e a gostar de esporte. Neste processo, existem as relações sociais, os valores, princípios que se desenvolvem e se manifestam durante o processo de ensinar e aprender, dentro e fora do campo de jogo. Em meio a isso, se constitui uma história, um caminho que deve ser responsável, acima de tudo, pelo desenvolvimento humano. Encontramos na Pedagogia do Esporte uma forma de investigar e desenvolver o ensino e a educação por meio dos esportes e a nossa proposta é aplicá-la ao Tchoukball, um esporte coletivo que vem ganhando novos praticantes no Brasil e no exterior. Com base nos três Referenciais da PE. Objetivo: É responder a pergunta: Como ensinar Tchoukball? Com isso, visamos estruturar procedimentos pedagógicos para o ensino desta modalidade esportiva.

Palavras-chave: Tchoukball; Pedagogia do Esporte; Ensino. 


\title{
INFLUENCIA DE LAS VARIABLES PEDAGÓGICAS EN LA CARGA DE ENTRENAMIENTO EN BALONCESTO EN FORMACIÓN
}

\author{
Laura Cáceres-Sánchez \\ https://orcid.org/0000-0003-1499-7246 (iD) \\ Universidad de Extremadura (Cáceres - España) \\ lauracs@unex.es \\ Celia Sánchez-Menaya \\ https://orcid.org/0000-0003-0330-6665 (iD \\ Universidad de Extremadura (Cáceres - España) \\ celiasanchezmenaya@gmail.com
}

Antonio Antúnez

https://orcid.org/0000-0002-7451-4448

Universidad de Extremadura (Cáceres - España)

antunez@unex.es

Sergio José Ibáñez

https://orcid.org/0000-0001-6009-4086 (iD

Universidad de Extremadura (Cáceres - España)

sibanez@unex.es

Link do Painel:

https://www.youtube.com/watch?v=adgXr8DDuVU\&list=PLJQfgfDuqS1tDpTzZzgdLchBDvZa8SIAp\&index=10

\begin{abstract}
Resumo
Introducción. Un aspecto fundamental para asegurar el éxito deportivo es desarrollar entornos de entrenamiento adecuados. Una de las estrategias más empleadas para la optimización de las tareas de entrenamiento es modificar el número de jugadores, las situaciones de juego. Además, para una formación adecuada del jugador se requiere del entrenamiento de todos los contenidos relacionados con las diferentes fases de juego. Estas situaciones se pueden clasificar en small sided Games (de desigualdad o igualdad) o juegos reducidos, situaciones reales de juego o full games, situaciones sin oposición y situaciones individuales. Por ello, el objetivo de este trabajo fue analizar la influencia de la situación de juego y la fase de juego en variables de carga externa empleadas por un entrenador para la planificación de sus entrenamientos en un equipo de categoría infantil de baloncesto. Metodología. Se analizaron 91 tareas de entrenamiento de 15 sesiones de la temporada 2019/2020, registradas a través del Sistema Integral para el Análisis de las Tareas de Entrenamiento (SIATE). Se analizaron las variables pedagógicas fase de juego y situación de juego junto a las variables de carga externa del SIATE: grado de oposición, densidad de la tarea, $\mathrm{n}^{\circ}$ de ejecutantes simultáneos, carga competitiva, espacio de juego, implicación cognitiva, carga de la tarea, carga de participación y carga de la tarea por tiempo. Se realizó un análisis descriptivo e inferencial empleando las pruebas estadísticas chi cuadrado $\left(\chi^{2}\right), V$ de Cramer $(V C)$, residuos tipificados corregidos $(R T C)$ y el coeficiente de correlación de pearson ( $r$ ). Resultados y Discusión. Sobre la base a los resultados obtenidos en este estudio se concluye que las variables de carga externa están relacionadas y asociadas con la situación de juego, pues según aumenta el número de participantes, mayor es la intensidad y la complejidad de la tarea, siendo la carga de la tarea mayor. además, los resultados muestran la existencia de asociación estadísticamente significativas entre la fase de juego con las variables de grado de oposición, el número de ejecutantes simultáneos, carga competitiva e implicación cognitiva. Conclusiones. La carga teórica total de la tarea es mayor cuando se entrenan tareas defensivas o mixtas, pero cuando se pondera esta carga a la participación de los jugadores se invierte esta relación. Gracias a datos se puede mejorar el conocimiento del proceso de entrenamiento y con ello la intervención del entrenador.
\end{abstract}

Palabras clave: Tareas de Entrenamiento, Situación de Juego; Fase de Juego; Variables de Carga Externa.

\section{AGRADECIMIENTOS}

Trabajo parcialmente subvencionado por la Ayuda a los Grupos de Investigación (GR18170) del Gobierno de Extremadura (Consejería de Economía, Ciencia y Agenda Digital); con la aportación de la Unión Europea a través de los Fondos Europeos de Desarrollo Regional. 


\title{
INFLUENCIA DEL CAMBIO DE SISTEMA PUNTUACIÓN EN LA METODOLOGÍA DE ENTRENAMIENTO EN PATINAJE ARTÍSTICO
}

\author{
Celia Sánchez-Menaya \\ https://orcid.org/0000-0003-0330-6665 \\ Universidad de Extremadura (Cáceres - España) \\ celiasanchezmenaya@gmail.com \\ Laura Cáceres-Sánchez \\ https://orcid.org/0000-0003-1499-7246 \\ Universidad de Extremadura (Cáceres - España) \\ lauracs@unex.es
}

Antonio Antúnez

https://orcid.org/0000-0002-7451-4448 iD

Universidad de Extremadura (Cáceres - España)

antunez@unex.es

Sergio José Ibáñez

https://orcid.org/0000-0001-6009-4086 iD

Universidad de Extremadura (Cáceres - España)

sibanez@unex.es

Link do Painel:

https://www.youtube.com/watch?v=VeFMPWLwEtU\&list=PLJQfgfDuqS1tDpTzZzgdLchBDvZa8SIAp\&index=9

Resumo

Introducción. El patinaje artístico es una disciplina deportiva en continua evolución, sujeta a cambio reglamentario, en la que los patinadores ejecutan diferentes figuras, saltos, piruetas y giros. Engloba seis disciplinas: figuras obligatorias o escuela, libre, parejas, danza, show y precisión. La competición en modalidad libre está compuesta por dos programas: programa corto y programa largo; compuestos a su vez por una serie de elementos técnicos (puntuación A), combinados con habilidades artísticas (puntuación B), que deben ser ejecutados en un límite de tiempo, según indica el reglamento de la Real Federación Española de Patinaje (2019), aceptado por el Comité Internacional de Patinaje Artístico. El antiguo sistema de puntuación White utilizaba algoritmos para calcular las victorias de los participantes, así como para desempatarlos y obtener una clasificación final, en función del cálculo de cada juez y el cálculo White. Sin embargo, la nueva filosofía RollArt basa sus pilares en la calidad de los elementos ejecutados, según su dificultad, y de los componentes de la impresión artística, en base a criterios concretos y objetivos. Por ello, el objetivo del presente estudio fue analizar la influencia del cambio del sistema de puntuación en la metodología de entrenamiento en patinaje artístico. Metodología. El diseño siguió los procedimientos de una investigación empírica de metodología cuantitativa, bajo una estrategia asociativa, llevándose a cabo un estudio comparativo en el que se analizaron las variables independientes categoría, género y sistema de puntuación y las variables dependientes programa corto, programa largo y puntuación final, dentro de las cuales se incluyen los componentes técnico y artístico de esta disciplina. La muestra estuvo constituida por 98 patinadores, 52 junior y 46 senior. La actuación de cada patinador fue analizada bajo el sistema de puntuación White y la filosofía RollArt. Para el análisis de diferencias entre las puntuaciones obtenidas y la clasificación del campeonato se utilizó la prueba no paramétrica de los rangos con signo de Wilcoxon, mientras que para la identificación de relaciones entre ambos sistemas de puntuación se utilizó el coeficiente de correlación de Spearman. Resultados y Discusión. A partir de los resultados obtenidos, fue posible afirmar la existencia de diferencias significativas entre ambos sistemas de puntuación, hecho que aporta información relevante para técnicos y patinadores, permitiéndoles conocer qué aspectos les proporcionarán mayor puntuación, orientando así los procesos de entrenamiento de manera adecuada. Conclusiones. Los resultados fruto del análisis realizado concluyen en que las puntuaciones obtenidas bajo el sistema de puntuación son más altas, a pesar de que ambos sistemas estén relacionados. Este hecho hace que entrenadores y patinadores modifiquen su visión sobre los procesos de entrenamiento, siendo necesario plantear una nueva metodología que aproveche la información relevante que los datos de competición les proporcionan, pues les permite conocer qué aspectos les otorgarán una mayor puntuación en los campeonatos, así como las directrices sobre su distribución y enlace con el componente artístico.

Palabras clave: Patinaje Artístico; Sistema de Puntuación; Metodología de Entrenamiento. 
Agradecimientos

Trabajo parcialmente subvencionado por la Ayuda a los Grupos de Investigación (GR18170) del Gobierno de Extremadura (Consejería de Economía, Ciencia y Agenda Digital); con la aportación de la Unión Europea a través de los Fondos Europeos de Desarrollo Regional. 


\title{
AS POSSIBILIDADES E POTENCIALIDADES TÉCNICO-TÁTICAS DO JOGO/BRINCADEIRA DE BOBINHO EM JOVENS FUTEBOLISTAS
}

\author{
João Bosco Gomes Lima Júnior \\ https://orcid.org/0000-0001-8315-730X \\ http://lattes.cnpq.br/4802924119461331 \\ Universidade Estadual de Campinas (Campinas, SP - Brasil) \\ bosco_junior@outlook.com
}

Alberto Lobato Góes Júnior

https://orcid.org/0000-0002-7421-3901

http://lattes.cnpq.br/2963844434616048 9

Universidade Estadual de Campinas (Campinas, SP - Brasil)

algj1421@gmail.com

Alcides José Scaglia

http://orcid.org/0000-0003-1462-1783

http://lattes.cnpq.br/6052868681786447

Universidade Estadual de Campinas (Campinas, SP - Brasil)

alcides.scaglia@fca.unicamp.br

Link do Painel:

https://www.youtube.com/watch?v=7dvX98JDDTI\&list=PLJQfgfDuqS1tDpTzZzgdLchBDvZa8SIAp\&index=5

\section{Resumo}

Objetivo: O objetivo do estudo é verificar se diferentes jogos de bobinhos geram as mesmas possibilidades (conteúdo evidente) e potencialidades (conteúdo latente) técnico-táticas em jogadores. Metodologia: Participaram do estudo 28 jogadores (SUB-13; SUB-17), respeitando os procedimentos éticos. Aplicamos dois jogos: bobinho 01 (dois toques) e bobinho 02 (um toque), e utilizamos os indicadores técnico-táticos propostos por Scaglia et al. (2021) para analisá-los. Na análise estatística, realizamos o teste de Shapiro-Wilk para verificar a normalidade dos dados, posteriormente, realizamos o teste de Mann-Whitney para comparar os jogos, adotando o nível de significância de $5 \%(\mathrm{p}<0,05)$. O tratamento foi realizado no programa GraphPad Prism. Resultados: $O$ passe e o domínio surgiram como as principais possibilidade nas categorias. Nas potencialidades, o sub-13 interviram mais sobre a bola em bobinho 01, enquanto o sub-17 desempenhou-se melhor no jogo 01, em geral. Conclusão: Logo, as possibilidades são as mesmas independentemente da idade dos jogadores, porém as potencialidades são diferentes.

Palavras-chave: Possibilidades; Potencialidades; Bobinho; Futebol. 


\title{
O ENSINO-APRENDIZAGEM DO FUTSAL: UM OLHAR PARA A EDUCAÇÃO BÁSICA E O ENSINO SUPERIOR
}

\author{
Cesar Vieira Marques Filho \\ https://orcid.org/0000-0001-6654-8982 i \\ http://lattes.cnpq.br/8670985499726497 \\ Universidade Estadual de Campinas (Campinas, SP - Brasil) \\ cesarvmf@hotmail.com \\ Luís Felipe Nogueira Silva \\ https://orcid.org/0000-0003-0583-4445 \\ http://lattes.cnpq.br/0793380850491870 \\ Universidade Estadual de Campinas (Campinas, SP - Brasil) \\ luisfelipenogu@gmail.com \\ Larissa Rafaela Galatti \\ https://orcid.org/0000-0003-1743-6356 \\ http://lattes.cnpq.br/3409947437523352 \\ Universidade Estadual de Campinas (Campinas, SP - Brasil) \\ Igalatti@unicamp.br \\ Alcides José Scaglia \\ http://orcid.org/0000-0003-1462-1783 \\ http://lattes.cnpq.br/6052868681786447 \\ Universidade Estadual de Campinas (Campinas, SP - Brasil) \\ alcides.scaglia@fca.unicamp.br \\ Paulo Cesar Montagner \\ http://orcid.org/0000-0002-5764-8022 \\ http://lattes.cnpq.br/5260809428528760 \\ Universidade Estadual de Campinas (Campinas, SP - Brasil) \\ cesar.montagner@unicamp.br \\ Link do Painel:
}

https://www.youtube.com/watch?v=wWVKxRIsh0A\&list=PLJQfgfDuqS1tDpTzZzgdLchBDVZa8SIAp\&index=8

\begin{abstract}
Resumo
Introdução: Os processos de ensino-aprendizagem do futsal podem ser embasados por diferentes perspectivas e direcionados a contextos distintos. Neste estudo, propomos uma discussão deste cenário em relação às aulas de Educação Física Escolar e às disciplinas específicas da modalidade em cursos de graduação em Educação Física. Percebemos as particularidades no trato com o futsal, esporte amplamente difundido e praticado no território brasileiro. Os métodos de ensino e o aspecto social na formação dos praticantes aparecem como temas que geram importantes reflexões. Podemos traçar algumas aproximações entre o futsal praticado na escola e o ensinado nas universidades, tendo a Pedagogia do Esporte como pano de fundo para estabelecer tal discussão.
\end{abstract}

Palavras-chave: Futsal; Educação Básica; Ensino Superior. 


\title{
SISTEMA PARA EL ANÁLISIS LAS VARIABLES ORGANIZATIVAS, PEDAGÓGICAS Y METODOLÓGICAS EN EDUCACIÓN FISICA
}

\author{
Antonio Antúnez \\ https://orcid.org/0000-0002-7451-4448 \\ Universidad de Extremadura (Cáceres - España) \\ antunez@unex.es \\ Juan de la Cruz \\ Universidad de Extremadura (Cáceres - España) \\ jhernandy@alumnos.unex.es \\ Sergio José Ibáñez \\ https://orcid.org/0000-0001-6009-4086 \\ Universidad de Extremadura (Cáceres - España) \\ sibanez@unex.es \\ Sebastián Feu \\ https://orcid.org/0000-0003-2959-5960 (iD \\ Universidad de Extremadura (Cáceres - España) \\ sfeu@unex.es \\ Link do Painel:
}

https://www.youtube.com/watch?v=X_iW53aqzpl\&list=PLJQfgfDuqS1tDpTzZzgdLchBDvZa8SIAp\&index=4

\begin{abstract}
Resumo
Introdução. El análisis de tareas de Educación Física es fundamental para la optimización de los procesos de enseñanza y formación del alumnado y para ello proponemos el Sistema Integral de Análisis de Tareas de Educación Física (SIATEF) como instrumento que plantea el registro y análisis de i) las variables organizativas, para obtener niveles óptimos de aprendizaje con una adecuada organización de la clase en aspectos relacionados la optimización del tiempo de aprendizaje efectivo, el espacio, la forma de participación, los materiales y recursos didácticos; ii) las variables pedagógicas, aquellas que ofrecen información al docente sobre las características de la tarea, contenido que se desea trabajar, tipo de actividad, su relación entre los aprendizajes, y explican cómo se estructura la tarea; y iii) las variables metodológicas que intervienen en el proceso de enseñanza-aprendizaje. Este trabajo describe el SIATEF como herramienta para analizar las tareas que se desarrollan en las sesiones de educación física con el fin de contribuir al desarrollo profesional del profesorado de Educación Física en el ámbito educativo. Metodologia, Resultados y Discusión. La elaboración de la herramienta se plantea como un estudio instrumental destinado al diseño y estudio de las propiedades psicométricas de instrumentos de medición de forma válida y fiable. La selección de la muestra de expertos que validaría el instrumento se realizó de forma deliberada e intencional, ajustándose a los criterios establecidos por los investigadores, además de tener en cuenta la accesibilidad de los mismos. Los expertos valoraron cuantitativamente la univocidad, pertinencia e importancia de cada categoría. Los resultados obtenidos mostraron un instrumento válido y fiable para el análisis de la planificación y la intervención del docente de educación física obteniendo niveles óptimos de validez de contenido y de consistencia interna. Los resultados del análisis de la fiabilidad a través del coeficiente de $\alpha$ de Cronbach para las variables Univocidad $(\alpha=.94)$, Pertinencia $(\alpha=.89)$, e importancia ( $\alpha=.92$.), se consideran excelentes. Conclusión. En conclusión, el SIATEF presenta una alta validez de contenido y fiabilidad y puede ser empleado para el análisis de las tareas en Educación Física. Es un instrumento que puede ser empleado tanto por investigadores como por docentes, pudiendo realizar valoraciones sobre las diferencias que se produzcan entre lo que se planifica para la clase y lo que sucede en la misma. Esta herramienta puede constituirse en un punto de partida para la reflexión del docente tanto en fase de pre-servicio como en servicio.
\end{abstract}

Palabras clave: Tareas de Educación Física; Enseñanza y Formación del Alumnado; SIATEF. 


\title{
VARIABILIDAD DE LA CARGA EXTERNA E INTERNA ATENDIENDO A LA SITUACIÓN DE JUEGO EN EL DESEMPEÑO DEL FÚTBOL ESCOLAR
}

\author{
Juan Manuel García-Ceberino \\ https://orcid.org/0000-0002-2922-4935 (iD \\ Universidad de Extremadura (Cáceres - España) \\ jmanuel.jmgc@gmail.com \\ María Graças Gamero \\ Universidad de Extremadura (Cáceres - España) \\ mgamerob@alumnos.unex.es
}

Sebastián Feu

https://orcid.org/0000-0003-2959-5960

Universidad de Extremadura (Cáceres - España)

sfeu@unex.es

Link do Painel:

https://www.youtube.com/watch?v=mSAGDFmtfNO\&list=PLUGgfDuqS1tDpTzZzgdLchBDvZa8SIAp\&index=7

\section{Resumo}

Introdução. El deporte del fútbol tiene un gran potencial para que los escolares realicen actividad física muy exigente. Así, los profesores de Educación Física, al diseñar las sesiones de este deporte, deben prestar atención a diferentes condicionantes de las tareas (p.ej. la Situación de Juego, SJ) para provocar elevadas exigencias físicas (carga externa) y fisiológicas (carga interna). El objetivo del estudio fue identificar que situaciones de juego utilizadas en el diseño de diferentes tareas de aprendizaje del fútbol provocan mayores exigencias físico-fisiológicas. Método. Estudio cuasi-experimental y transversal. Participaron un total de 41 escolares, con edad comprendida entre 10 y 11 años ( $5^{\circ}$ nivel de Educación Primaria), de un colegio público de España. Los escolares practicaron distintas tareas de aprendizaje del fútbol durante 11 sesiones. Éstos fueron equipados con dispositivos inerciales WIMU ProTM (RealTrack System, Almería, España) colocados en un arnés anatómico, y monitores de frecuencia cardiaca-FC. La unidad de análisis fue el registro de los escolares en cada tipo de tarea ejecutada durante las sesiones. La variable independiente del estudio fue la SJ, agrupada en cinco categorías: tareas sin oposición (1×0...); juego individual y

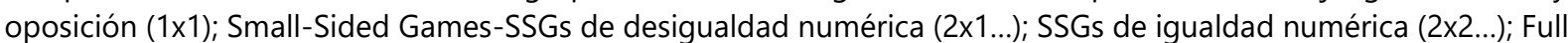
Games-FGs (5x5). Las variables dependientes fueron: Player Load por minuto (PL/min, variable neuromuscular de carga externa); y $\mathrm{FC}$ media ( $\mathrm{FC}_{\text {avg, }}$ variable de carga interna). Resultados y discusión. La prueba Kruskal-Wallis $\mathrm{H}$ señala diferencias significativas en las variables estudiadas $\left(\mathrm{PL} / \mathrm{min}\right.$ y $\mathrm{FC}_{\text {avg }}$ ), según la SJ. Igualmente, las comparaciones múltiples por parejas expresan diferencias significativas a favor de los SSGs de igualdad numérica y los FGs. No hay diferencias entre ambas SJ. En este sentido, Póvoas et al. (2018) obtuvieron que a pesar de que los SSGs provocan mayor intensidad que los FGs $(p<0.05)$, ambas SJ registran elevados niveles de intensidad. A diferencia de nuestro estudio ( $5 \times 5$ como FGs), los autores mencionados categorizaron la SJ 12x12. De forma que, las diferencias significativas resultantes podrían deberse al mayor uso de sujetos, provocando un menor contacto con el balón. Además, las tareas descontextualizadas y aisladas del juego real, con ausencia de oponentes, alcanzan menor intensidad que las tareas con oposición. Conclusión. Los escolares cuando se agrupan en SSGs de igualdad numérica y FGs se mueven/desplazan más (carga externa) y, además, la intensidad de estas tareas de aprendizaje del fútbol es elevada (carga interna). Se recomienda el uso de ambas SJ en el desempeño del fútbol en Educación Física para lograr niveles adecuados de actividad física, asociados con mejoras cardiovasculares y de salud.

Palabras clave: Situaciones de Juego; Tareas de Aprendizaje; Demandas Físico-Fisiológicas, Fútbol Escolar WIMU ProTM.

\section{Agradecimientos}

Trabajo parcialmente subvencionado por la Ayuda a los Grupos de Investigación (GR18170) del Gobierno de Extremadura (Consejería de Economía, Ciencia y Agenda Digital); con la aportación de la Unión Europea a través de los Fondos Europeos de Desarrollo Regional. 


\title{
CURRÍCULO DA FORMAÇÃO DE JOVENS FUTEBOLISTAS: A INICIAÇÃO ESPORTIVA
}

\author{
Elson Aparecido de Oliveira \\ https://orcid.org/0000-0001-5652-7109 iD \\ http://lattes.cnpq.br/2109830550356968 \\ Centro Universitário de Várzea Grande (Várzea Grande, MT - Brasil) \\ elson.oliveira@univag.edu.br \\ Riller Silva Reverdito \\ https://orcid.org/0000-0003-0556-9151 iD \\ http://lattes.cnpq.br/3357837391641002 9 \\ Universidade do Estado de Mato Grosso (Cáceres, MT - Brasil) \\ rsreverdito@unemat.br \\ Link do Painel:
}

https://www.youtube.com/watch?v=_q75qkxoJzl\&list=PLJQfgfDuqS1uvgh0dSweYcCyGhruByGXM\&index=5

\section{Resumo}

Introdução: Os cenários que ofertam iniciação ao futebol recebem milhões de jovens. Contudo, a iniciação ainda ocorre de forma pouco sistematizada e organizada em se tratando de um percurso de formação. Objetivo: Analisar a percepção de especialistas no futebol sobre elementos e possibilidades para uma matriz curricular na iniciação do futebol. Metodologia: Foram ouvidos por meio de entrevista semiestruturada cinco líderes de grupos de pesquisa de futebol, registrados no Diretório de Grupos de Pesquisa Lattes CNPq. Resultados: Os resultados apontam multiplicidade de possibilidades para a matriz curricular. Os pontos convergentes sinalizam para um currículo que pode ter objetivos amplos e específicos relacionados à formação humana e esportiva, contemplando um conjunto de objetivos e conteúdos, habilidades e/ou competências a serem desenvolvidos pelos treinadores. Consideração: Conclui-se que ensinar futebol na iniciação passa por um currículo, que deve se desenvolver visando atingir objetivos de formação, os quais devem ser definidos para balizar a atuação dos treinadores (as).

Palavras-chave: Iniciação Esportiva; Futebol; Pedagogia do Esporte. 


\title{
DESENVOLVIMENTO DE ATLETAS: A INFLUÊNCIA DA QUALIDADE DA DINÂMICA SOCIAL
}

\author{
Leilane Alves de Lima \\ http://orcid.org/0000-0002-7866-4152 ip \\ http://lattes.cnpq.br/5949245089655580 \\ Universidade Estadual de Campinas (Campinas, SP - Brasil) \\ leilane.alima@gmail.com \\ Larissa Rafaela Galatti \\ https://orcid.org/0000-0003-1743-6356 \\ http://lattes.cnpq.br/3409947437523352 \\ Universidade Estadual de Campinas (Campinas, SP - Brasil) \\ Igalatti@unicamp.br \\ Link do Painel:
}

https://www.youtube.com/watch?v=0MIRi_GKHvU\&list=PLJQfgfDuqS1uvgh0dSweYcCyGhruByGXM\&index=3

\begin{abstract}
Resumo
Introdução: A dinâmica das interações sociais tem sido amplamente apontada como elemento importante para incitar a adesão voluntária à prática esportiva. Porém, possuímos poucas informações quanto sua influência na carreira esportiva de atletas de elite. Objetivo: Compreender a influência da dinâmica social no engajamento e continuidade na prática esportiva. Metodologia: Pesquisa retrospectiva desenvolvida com sete atletas campeãs mundiais pela Seleção Brasileira de Handebol feminino. Os dados foram coletados através de entrevistas semiestruturadas, os quais foram transcritos e analisados através dos procedimentos contidos na análise temática. Resultado Representada através da influência positiva da família, treinadores e amigos/colegas, a qualidade da dinâmica social se manifestou como elemento mais significativo ao longo da carreira, permitindo que as atletas superassem dificuldades estruturais, financeiras e culturais. Além disso, facilitou na adaptação e engajamento em diferentes contextos. Conclusão. Na percepção das atletas, a qualidade da dinâmica social foi um elemento fundamental para continuidade na prática esportiva.
\end{abstract}

Palavras-chave: Elementos Dinâmicos; Carreira Esportiva; Handebol Feminino. 


\title{
DO ESPORTE PARA A VIDA CONTRIBUIÇÕES DA PEDAGOGIA DO ESPORTE E DO DESENVOLVIMENTO POSITIVO DE JOVENS PARA A FORMAÇÃO DO CIDADÃO
}

\author{
Artur Goulart Berger \\ https://orcid.org/0000-0003-3691-6873 \\ http://lattes.cnpq.br/4962672195040292 \\ Universidade Federal do Rio Grande do Sul (Porto Alegre, RS - Brasil) \\ artur.berger@ufrgs.br \\ Thiago José Leonardi \\ https://orcid.org/0000-0002-3843-2648 \\ http://lattes.cnpq.br/7904396285319903 9 \\ Universidade Federal do Rio Grande do Sul (Porto Alegre, RS - Brasil) \\ thiago.leonardi@ufrgs.br \\ Link do Painel:
}

https://www.youtube.com/watch?v=4FFDnxWirtA\&list=PLJQfgfDuqS1uvgh0dSweYcCyGhruByGXM\&index=4

\section{Resumo}

Objetivo: Este estudo teve como objetivo identificar e descrever como o esporte pode ser utilizado como meio para a formação do cidadão. Metodologia: Partindo dos conceitos teóricos da Pedagogia do Esporte, por meio das técnicas de pesquisa de revisão narrativa e sistemática da literatura, dialogamos e buscamos interfaces com o Desenvolvimento Positivo de Jovens. Identificamos que a literatura da Pedagogia do Esporte apresenta uma base teórica consistente acerca do tratamento intencional dos conteúdos socioeducativos, porém, carece de evidências empíricas relacionadas ao ensino-vivência-aprendizagem destas questões. Diante do exposto, abordamos inicialmente a possibilidade de diálogo com a literatura do Desenvolvimento Positivo de Jovens, e, identificando a convergência entre os princípios básicos de ambas as abordagens, olhamos para os estudos de intervenção realizados com objetivos formativos. Assim, destacamos as decisões metodológicas que potencializaram as discussões desenvolvidas nestes estudos, indicando possíveis caminhos para futuras pesquisas na Pedagogia do Esporte.

Palavras-chave: Esporte; Pedagogia do Esporte; Desenvolvimento Positivo de Jovens; Formação do Cidadão; Valores. 


\title{
ESPORTE NO CONTEXTO ESCOLAR: CENÁRIO DAS PUBLICAÇÕES EM PERIÓDICOS BRASILEIROS DE 1990 A 2019
}

\author{
Edésio Rodrigues da Silva Júnior \\ https://orcid.org/0000-0003-2009-7359 iD \\ http://lattes.cnpq.br/3429623676073455 \\ Universidade Federal de Mato Grosso (Cuiabá, MT - Brasil) \\ ederodriguesjr@gmail.com \\ Riller Silva Reverdito \\ https://orcid.org/0000-0003-0556-9151 iD \\ http://lattes.cnpq.br/3357837391641002 9 \\ Universidade do Estado de Mato Grosso (Cáceres, MT - Brasil) \\ rsreverdito@unemat.br \\ Link do Painel:
}

https://www.youtube.com/watch?v=aPmPxa2bf_8\&list=PLJQfgfDuqS1uvgh0dSweYcCyGhruByGXM\&index=2

\begin{abstract}
Resumo
Introdução: O interesse pelo esporte escolar ampliou de forma expressiva nas últimas décadas. Objetivo: Apresentar um panorama do que vem sendo produzido sobre o esporte no contexto escolar, tendo como foco as produções em artigos científicos publicados em periódicos nacionais na área de Educação física e Esportes. Metodologia: Utilizamos como fonte dessa investigação dez periódicos, no recorte temporal de 1990-2019. Os artigos foram classificados em: -esporte nas aulas de educação física escolar, -esporte fora das aulas de educação física escolar (atividade extracurricular da escola) e -formação de professores no esporte escolar. Resultados: Os dados revelam um crescente interesse sobre o esporte no contexto escolar ao longo dos anos. Identificou-se que a maioria dos artigos foram publicados na última década e a maior incidência está relacionada ao esporte nas aulas de educação física escolar. Além do aumento na produção, observa-se um maior interesse em promover o desenvolvimento do esporte no contexto escolar.
\end{abstract}

Palavras-Chave: Esporte; Escola; Jovens Atletas; Pedagogia do Esporte.

Agradecimentos: Fundação de Amparo à Pesquisa do Estado de Mato Grosso (FAPEMAT), Rede Cedes Mato Grosso. 


\title{
GESTÃO EM PEDAGOGIA DO ESPORTE: UM ESTUDO DE CASO EM PROJETO SOCIAL ESPORTIVO
}

\author{
Eduardo Roberto Uhle \\ https://orcid.org/0000-0002-1617-2400 \\ http://lattes.cnpq.br/3856204227557114 \\ Universidade Estadual de Campinas (Campinas, SP - Brasil) \\ eduardo.uhle@gmail.com \\ Larissa Rafaela Galatti \\ https://orcid.org/0000-0003-1743-6356 \\ http://lattes.cnpq.br/3409947437523352 9 \\ Universidade Estadual de Campinas (Campinas, SP - Brasil) \\ lgalatti@unicamp.br \\ Link do Painel:
}

https://www.youtube.com/watch?v=3KgAXObeZvY\&list=PLQfgfDuqS1uvgh0dSweYcCyGhruByGXM\&index=6

\section{Resumo}

Introdução: Este estudo de caso exploratório- descritivo é resultado de uma pesquisa de mestrado sobre a gestão pedagógica do programa esportivo de uma ONG. Objetivo: foi desvelar como ocorreu a concepção e o desenvolvimento do programa à luz da pedagogia do esporte e os processos de gestão. Metodologia: Realizamos entrevistas, análise documental e observação direta, com registros realizados digitalmente e diário de campo. $\mathrm{Na}$ análise dos dados realizamos análise temática e consideramos o modelo Long Term Program Development. Resultado: Observamos que conhecer o contexto em que atua e considerá-lo na construção coletiva do programa possibilita o alinhamento e compartilhamento de propósito, fundamental para atuação em regiões de vulnerabilidade. Com relação à sustentação do programa, o acompanhamento próximo da equipe gestora, processos de desenvolvimento de comunidade de aprendizagem são pontos importantes. Por fim, identificamos uma parceria estratégica com outra ONG, complementa a carga horária dos educadores, gerando vínculos mais fortes com o programa.

Palavras-chave: Esportes; Pedagogia; Organizações Não-governamentais; Gestão Esportiva. 


\title{
DESENVOLVIMENTO DE HABILIDADES PARA A VIDA POR MEIO DO RUGBY
}

\author{
Vitor Ciampolini \\ http://orcid.org/0000-0003-2278-7310 \\ http://lattes.cnpq.br/9286890586590825 9 \\ Universidade Federal de Santa Catarina (Florianópolis, SC - Brasil) \\ vciampolini@gmail.com \\ Juarez Vieira do Nascimento \\ https://orcid.org/0000-0003-0989-949X iD \\ http://lattes.cnpq.br/0876604605174484 9 \\ Universidade Federal de Santa Catarina (Florianópolis, SC - Brasil) \\ juarez.nascimento@ufsc.br \\ Michel Milistetd \\ https://orcid.org/0000-0003-3359-6878 \\ http://lattes.cnpq.br/3003314961521718 \\ Universidade Federal de Santa Catarina (Florianópolis, SC - Brasil) \\ michel.milistetd@ufsc.br
}

Link do Painel: https://www.youtube.com/watch?v=Ziautw-OwJg\&list=PLJQfgfDuqS1uvgh0dSweYcCyGhruByGXM

\begin{abstract}
Resumo
Introdução: O desenvolvimento de habilidades para a vida é um processo complexo de desenvolvimento humano que vem ganhando destaque nas áreas da Psicologia e Pedagogia do Esporte. O rugby é uma modalidade esportiva que parece contar com algumas características que influencia diretamente neste processo, como os valores da modalidade e a cultura do amadorismo. Objetivo: Analisar o desenvolvimento de habilidades para a vida de exatletas de rugby. Metodologia: Por meio de uma abordagem qualitativa, este estudo de casos múltiplos irá investigar a trajetória de desenvolvimento de dois ex-atletas de rugby. De modo complementar, serão entrevistados uma série de agentes (pessoas próximas aos ex-atletas) que contribuirão para a riqueza dos dados. Entrevistas serão utilizadas como o principal procedimento de coleta. Resultado esperado: Espera-se que, por meio da análise temática dos dados, este estudo amplie a compreensão sobre os fatores que contribuem neste processo de desenvolvimento.
\end{abstract}

Palavras-chave: Desenvolvimento Humano; Habilidades Socioemocionais; Esporte; Qualitativo. 


\section{FÓRUM INTERNACIONAL DE PEDAGOGIA DO ESPORTE}

\section{Realização}

- Universidade Estadual de Campinas (UNICAMP)

- Universidade do Estado de Mato Grosso (UNEMAT)

\section{Grupos de Pesquisa}

- Grupo de Estudos em Pedagogia do Esporte - GEPESP/ UNICAMP

- Laboratório de Estudos Aplicados em Pedagogia do Esporte - LEAPE/ UNEMAT

- Laboratório de Estudos em Pedagogia do Esporte - LEPE/ UNICAMP
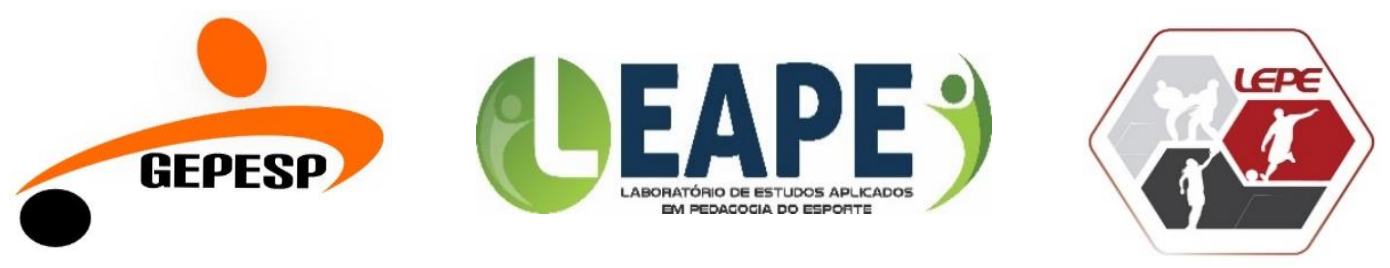

\section{Apoio ao Fórum}

- Programa Rede Cedes Mato Grosso - Centro de Desenvolvimento de Pesquisa em Políticas de Esporte e de Lazer da Rede Cedes. Ministério da Cidadania, Secretaria Especial do Esporte.

- Centro Interdisciplinar de Pesquisas em Esporte e Exercício Físico - CIPEEF/ Universidade do Estado de Mato Grosso - UNEMAT.

- Fundação de Amparo à Pesquisa do Estado de Mato Grosso - FAPEMAT.

- Fundação de Amparo à Pesquisa do Estado de São Paulo - FAPESP. 


\section{EQUIPE EDITORIAL}

\section{Comissão Organizadora}

- Prof. Dr. Roberto Rodrigues Paes

- Prof. Dr. Riller Silva Reverdito

- Profa. Dra. Larissa Rafaela Galatti

- Prof. Dr. Alcides José Scaglia

- Profa. Drda. Paula Simarelli

\section{Comissão Científica}

- Prof. Dr. Alcides José Scaglia

- Prof. Dr. Antonio Antúnez

- Prof. Dr. Heitor Andrade Rodrigues

- Profa. Dra. Larissa Rafaela Galatti

- Prof. Dr. Michel Milistetd

- Prof. Dr. Roberto Rodrigues Paes

- Prof. Dr. Riller Silva Reverdito

- Prof. Dr. Sebastián Feu

- Prof. Dr. Sergio José Ibáñez

- Prof. Dr. Thiago José Leonardi

\section{Comissão de Apoio}

- Bernardo dos Santos

- Edésio Rodrigues da Silva Junior

- Eduardo Dell Osbel

- Elson Aparecido de Oliveira

- Igor Oliveira dos Santos

- Karyna Aires da Silva

- Leilane Alves de Lima

- Luciano Alves Santos

- Matheus Grandim de Almeida

- Rafael Pascutti Ferreira

- Silas Sinotti

- Stefany Caroliny de Souza

- Talita Maria da Silva

- Uissara Tainá Silvério dos Santos 